\title{
Modeling For Predicting Strength of Carbon Nanostructures
}

\author{
Sangwook Sihn ${ }^{\mathrm{a}, \mathrm{b}, *}$, Vikas Varshney ${ }^{\mathrm{a}, \mathrm{c}}$, Ajit K. Roy ${ }^{\mathrm{a}}$, Barry L. Farmer ${ }^{\mathrm{a}}$ \\ ${ }^{a}$ Air Force Research Laboratory, Materials and Manufacturing Directorate \\ AFRL/RXAN, Wright-Patterson AFB, OH 45433, USA \\ ${ }^{b}$ University of Dayton Research Institute, Multi-Scale Composites and Polymers Division, \\ 300 College Park, Dayton, OH 45469-0060, USA \\ ${ }^{c}$ Universal Technology Corporation, 1270 N. Fairfield Rd., Dayton, OH 45432, USA
}

\section{Abstract}

We have developed a computational scheme to predict stiffness and strength of carbon nanostructures under various loading modes. The prediction method is based on combined molecular mechanics and molecular dynamics simulations to ensure a global energy minimum at a given loading level. We have applied the present method to various carbon nanostructures including carbon nanotubes (CNTs), graphene, CNT with defects, a CNT-graphene junction and pillared graphene nanostructures. For all cases, we have identified the maximum stress and strain at failure of these carbon nanostructures as well as their critical failure modes, and discussed mechanisms that lead to their catastrophic failure.

\footnotetext{
*Corresponding author: Tel: +1-937-255-9067.

Email address: sangwook@alumni.stanford.edu (Sangwook Sihn)
} 


\section{Background and Introduction}

With increasing needs for better efficiency, more and more modern structural systems, satellites and space structures require multi-functionality having not only structural integrity but also thermal and electrical performance. Many previous studies have shown that carbon-based molecular structures such as diamond, carbon nanotube (CNT) and graphite possess superior multifunctional properties including stiffness, strength, thermal and electrical conductivities because of their ordered structures as well as strong carboncarbon (C-C) bonds. There have been numerous efforts to enhance the performance of the composite structures and materials by taking advantage of these superior multi-functional properties of the carbon nanostructure [1-3].

CNTs and graphite are inherently 1-D and 2-D structures, and hence, their physical properties are highly anisotropic in nature. Recently, many efforts have been made to fabricate more advanced carbon nanomaterials by having 3-D junctioned structures with the CNTs and the graphene layers $[4-7]$. One of such structures is a pillared graphene nanostructure (PGN), which can be fabricated by growing CNT forests on graphene/graphite layers. The 3-D carbon nanostructures have drawn much attention lately among researchers because of their superior multi-dimensional functionality, which are difficult to achieve with pure 1-D CNTs or 2-D graphene and graphite structures $[8,9]$.

In order for these carbon nanostructures to be used as reinforcing constituents in the advanced composite materials and structures, one needs to assess their structural performance subject to various loading conditions. Such structural performance can be evaluated by mechanical properties such 
as stiffness, Poisson's ratio [9], maximum stress and strain to failure, etc. Among numerical analytical works to predict the mechanical properties of the carbon-based molecular architectures, widely and promisingly used are the atomistic modeling, such as molecular dynamics (MD) simulations [10, 11], tight binding MD [12], density functional theory [13], classical continuum

mechanics [14-16] and structural mechanics approach [17-19]. While many aspects of the carbon nanostructures have been discussed in literature, only a few studies have been conducted to predict their mechanical strength subject to various modes of loading such as tension, compression and shear loads, especially for the complicated 3-D carbon nanostructures [20-24].

In the current study, we have developed a computational methodology based on identification of global minimums to predict the mechanical strength of various carbon nanostructures, such as CNTs, graphene sheets, CNTgraphene and CNT-CNT junctioned nanostructures, and have identified their critical failure modes. The details of the simulation methodology are described in the following section. We have applied the current method to predict the maximum load-carrying capability of various carbon nanostructures as well as their damage behavior.

\section{Simulation Methodology}

The method used in the strength prediction of various carbon nanostructures is based on combined molecular mechanics (MM) and molecular dynamics (MD) simulation schemes. All simulations were conducted with molecular simulation software, LAMMPS [25]. As will be discussed below, the MM and MD schemes ensure the realization of globally minimal energy 
configuration of the carbon nanostructures at a given loading and boundary conditions.

The strength prediction simulation starts with an initial configuration of a nanostructure by applying a small increment of the strain (typically 0.01$0.1 \%$ strain) in a desired loading direction. Other portions of carbon atoms are constrained by prescribed boundary conditions depending on modes of loading. The increment of the applied strain results in increase of an interatomic potential energy, while the kinetic energy remains unchanged under an assumption of static loading. We conducted the MM simulations to find the minimal energy configuration subject to increased interatomic potential at each loaded stage.

General interatomic potentials of various carbon nanostructures based on $\mathrm{sp}^{2}$ hybridized bonds can be written as a sum of all possible potential energy components as

$$
E=E_{b}+E_{\theta}+E_{\phi}+E_{\omega}+E_{\nu}
$$

where $E_{b}, E_{\theta}, E_{\phi}, E_{\omega}$ and $E_{\nu}$ are the potential energy terms due to bond stretching, angle bending, dihedral angle twisting, out-of-plane bending and non-bond van der Waals (VDW) interactions, respectively. We ignored other non-bond potential terms such as an electrostatic energy term as studied systems are single-atom type neutral systems. In the present study, we used a modified Morse potential function for the bond stretching as

$$
E_{b}=D_{e}[1-\exp (-\beta \Delta r)]^{2},
$$

where $\Delta r=r-r_{o}$, and $r$ and $r_{o}$ are distances between two bonded atoms at current and original stages, respectively. The parameters used in Eq. 2 for 
carbon-carbon bond are $D_{e}=86.8 \mathrm{kcal} / \mathrm{mol}, \beta=2.625 \AA^{-1}$ and $r_{o}=1.421$ $\AA[20]$. For the angle bending, we used a harmonic potential

$$
E_{\theta}=\frac{1}{2} k_{\theta}\left(\theta-\theta_{o}\right)^{2}
$$

where $\theta$ and $\theta_{o}$ are bond angle formed by three adjacent atoms at current and original stages, respectively. The parameters used in Eq. 3 for C-C sp ${ }^{2}$ bond are $k_{\theta}=130 \mathrm{kcal} / \mathrm{mol}_{-} \mathrm{rad}^{2}$ and $\theta_{o}=2.094 \mathrm{rad}$ [20]. For the dihedral angle twisting and out-of-plane bending, we used the following potentials:

$$
\begin{aligned}
& E_{\phi}=k_{\phi}(1-\cos 2 \phi), \\
& E_{\omega}=k_{\omega}(1-\cos 2 \omega),
\end{aligned}
$$

where $\phi$ and $\omega$ are the dihedral torsional angle and out-of-plane bending angle formed by four atoms at the current stage, respectively. The force constants used in Eqs. 4 and 5 are $k_{\phi}=40 \mathrm{kcal} / \mathrm{mol}_{-\mathrm{rad}^{2}}$ and $k_{\omega}=0.37 \mathrm{kcal} / \mathrm{mol}$ $\operatorname{rad}^{2}[26]$. For the non-bond VDW interaction, we used a 12-6 Lennard-Jones potential:

$$
E_{\nu}=4 \varepsilon\left[\left(\frac{\sigma}{r}\right)^{12}-\left(\frac{\sigma}{r}\right)^{6}\right] \text { for } r<r_{c}
$$

where $r$ is the distance between interacting atoms, $\varepsilon$ is the depth of the potential well, $\sigma$ is the distance at which the inter-molecular potential between the two atoms switches between attractive and repulsive interactions, and $r_{c}$ is the cutoff distance. The parameters used in Eq. 6 are $\varepsilon=0.0556 \mathrm{kcal} / \mathrm{mol}$, $\sigma=3.4 \AA$ and $r_{c}=10 \AA[20]$.

Since the molecules are to be loaded under static loading conditions, we only consider the potential energy and ignore the kinetic energy. Therefore, 
we have a single function of the potential energy to minimize with $3 N$ independent variables, which are the 3 components of the coordinates $(x, y$ and $z$ ) of $N$ atoms in the system. When the set of atoms are loaded at the boundaries, the whole system of atoms is repositioned with the minimal energy configuration. We used a nonlinear conjugate gradient method to find the equilibrium configuration of the atoms of the minimal energy. The conjugate gradient method requires evaluation of gradient (derivatives) of the potential energy with respect to a coordinate system. Denote that the gradient of the potential energy with respect to a position vector $\left(\mathbf{r}_{i}\right)$ of an atom $i$ is a force vector, which can be written as

$$
\mathbf{F}\left(\mathbf{r}_{i}\right)=-\nabla_{\mathbf{r}_{i}} E=\frac{\partial E}{\partial x_{i}} \mathbf{i}+\frac{\partial E}{\partial y_{i}} \mathbf{j}+\frac{\partial E}{\partial z_{i}} \mathbf{k}, \quad(i=1,2, \cdots, N),
$$

where $\left(x_{i}, y_{i}, z_{i}\right)$ are the Cartesian coordinate pair of the position vector $\mathbf{r}_{i}$, and $\mathbf{i}, \mathbf{j}$ and $\mathbf{k}$ are unit vectors in $x-, y$ - and $z$-directions, respectively. By using a chain rule, we can obtain the derivative of potential energy with respective to the Cartesian coordinate components. The detail derivations and formulae for the derivative terms of all potential energy components in Eq. 1 can be found in [27].

With a load increase at the boundary box, we calculate the net force vector $(\mathbf{F})$ acting on each atom based on the bond length, bending angle, etc. The net force applied on the atoms in turn alters the bond length, bending angle, etc., and thus needs to be updated according to the new atom configurations. Multiple iterations using the MM simulations are needed to update the force and the atom configuration until $\mathbf{F}$ reaches to zero for all atoms, which is a state of minimal potential energy. The iterative MM scheme was implemented in the LAMMPS software in obtaining the equilibrium config- 
uration of the atoms at the minimal energy stage. Upon finding equilibrium configuration at the given strain-loading stage, all six components of overall stresses on the systems were determined by a pressure-calculation scheme implemented in LAMMPS [28]. With the stress and strain components known, we obtained the stress-strain behavior of the carbon nanostructures with the load increase for various studied cases.

In conducting the MM simulations for searching the minimal energy configuration, the numerical scheme implemented in LAMMPS software does not guarantee the global minimum of the total potential energy states. Therefore, with complicated atomic structures, the MM scheme often leads to a local energy minimum configuration, which would influence the damage initiation and subsequent progression resulting in unreliable prediction values. To circumvent this problem, we implemented an additional step in order to find the global energy configuration by coupling the MM scheme with the MD simulations under an NVE (microcanonical) ensemble.

Under this ensemble, the MD simulations are conducted with constant number of atoms, simulation volume and total energy. The total energy consists of the potential energy and kinetic energy. During MM simulation stage, which is static simulation, the kinetic energy is zero since the velocities of the atoms are zero. In the local energy minimum configuration found by the MM simulations using LAMMPS, the force components as a whole atomic structure are zero, but the force components with respect to individual degrees of freedom of the atoms are not zero. During the MD simulation stage, accelerations and velocities of the atoms are calculated based on these non-zero force components acting on the individual atoms, which results in 
nonzero kinetic energy. In effect, running the MD simulations effectively perturbs the system along the gradient directions by introducing the kinetic energy to the system. Since the NVE simulations ensure the total energy to remain constant, the increase in the kinetic energy results in reducing the potential energy by the same amount, which effectively results in jumping out of the local minimum and going down towards the global minimum direction. Consequently, by alternating the combined MM and MD simulations in an iterative way, we ensure to search the globally minimal energy state at a given loading step.

Figure 1 shows a flowchart that summarizes the present strength prediction scheme. Starting with the initial configuration of a nanostructure, a small increment of the strain is applied in a desired loading direction. While the increased strain is smaller than a preset maximum strain value, the MM simulation is conducted to find the minimal energy configuration using the Morse and other related potentials with respect to C-C bonds' deformation modes. Resulting stress and strain components are then calculated after the MM simulations. The system is then perturbed by the NVE MD simulations with a preset number of iterations. The combination of the MM and the MD simulations are repeated until the kinetic energy contribution to the total energy becomes negligibly small, and so do the force components acting on all the atoms. We evaluate the amount of kinetic energy in terms of a temperature of the system, and used the system temperature as an exit criterion. If the system temperature is larger than a preset tolerance temperature, $T_{t o l}$, the velocity components of all atoms are set to zero to suppress the kinetic energy contribution, and the MM simulations are conducted to 
obtain a new minimal energy state. When the system temperature is smaller than $T_{t o l}=10^{-6} \mathrm{~K}$, we consider that the kinetic energy contribution to the total energy becomes negligibly small, and thus exit the iteration loop of the MM and the MD combinational simulations for the current loading step. After increasing the strain, the combination of the MM and MD simulations are repeated to search the global energy minimum configuration at the new load step as discussed above.

\section{Results and Discussion}

We used the present method to predict the failure behavior and the stressstrain curves of various carbon nanostructures. The first case we considered is the graphene sheet under the tensile and shear loading. Stress-strain curves and deformed shapes of the graphene sheet stretched in two in-plane directions ( $x$ - and $y$-directions) are shown in Figures 2 and 3, and horizontally sheared is shown in Figure 4. Note that for visualization purpose, we plot the atom bonds with a cutoff distance of $0.16 \mathrm{~nm}$, and therefore, any bonds greater than this cutoff distance are not drawn.

As Figure 2 and Figure 3 show, under axial loading, the graphene sheet stretches uniformly along the loading direction and the axial stress increases monotonically with the increase of the strain loading until it reaches a maximum stress level. For the axial loading along $x$ - and $y$-directions, the maximum stresses are $\sigma_{1}=95.7 \mathrm{GPa}$ at $\varepsilon_{1}=15.7 \%$ strain, and $\sigma_{2}=120.3 \mathrm{GPa}$ at $\varepsilon_{2}=21.6 \%$, respectively. While the graphene sheet stretched in $x$-direction shows a drastic stress drop right after the maximum stress, the graphene sheet stretched in $y$-direction continues to sustain the maximum stress level 
until the drastic stress drop at $\varepsilon_{2}=22.2 \%$. The main reason of the drastic stress drop is that the graphene sheet is unable to stretch uniformly along the loading directions, so that some C-C bonds are stretched more than other bonds to maintain the minimal energy state. For the loading in $x$-direction, the longer stretch of bonds occurs along a vertical line perpendicular to the loading direction. For the loading in $y$-direction, the longer stretch occurs along a line perpendicular to the loading direction, but not in a straight way. For the shear load case, the stress increases monotonically with the increase of strain loading until at $\tau_{12}=154.0 \mathrm{GPa}$ and $\varepsilon_{12}=35.4 \%$, and drops drastically with further increase of shear loading.

We considered two different sizes of the graphene sheets $(2.56 \mathrm{~nm}$ by $2.95 \mathrm{~nm}$ denoted as $12 \times 12$ and $5.11 \mathrm{~nm}$ by $5.90 \mathrm{~nm}$ denoted as $24 \times 24)$ and compared the stress-strain behaviros under the three loading conditions. We generated a larger size $24 \times 24$ graphene sheet by duplicating the $12 \times 12$ one periodically in both $x$ - and $y$-directions. Figure 5 shows the comparison of the results. In this figure, dotted and solid lines are for $12 \times 12$ and $24 \times 24$ graphene sheets, respectively. The figure shows that the stress-strain behaviors of the two graphene sheets are nearly identical with each other for all three loading cases, which indicates that the stress-strain curves are relatively insensitive to the simulation size of the graphene sheets when the periodic boundary conditions are used.

The next case considered is the CNTs under tensile and compressive loading. The results for the four different chiralities of $(6,0),(6,6),(12,0)$ and $(12,12)$ CNTs are shown in Figure 6 . The figure shows that zigzag $(6,0)$ and $(12,0)$ CNTs yields lower failure stress and strain than armchair $(6,6)$ 
and $(12,12)$ CNTs. While the fractured surface of zigzag CNTs is nearly perpendicular to the loading direction, the fractured surface of the armchair CNTs has a slanted angle near $30^{\circ}$. For all CNTs, significant load drops occur after the catastrophic damage. We compared the stress-strain curves of the CNTs with those of the graphene sheets and plotted in Figure 7. In this figure, thick blurry lines are for graphene sheets loaded in $\sigma_{1^{-}}$and $\sigma_{2^{-}}$ directions. It can be seen that the stress-strain behaviors of both zigzag CNTs are similar with that of the graphene sheet loaded in $x$-direction, while those of both armchair CNTs are similar with that of the graphene sheet loaded in $y$-direction. The similar damage behaviors between the graphenes and CNTs suggest the significance of the loading direction with respect to the chirality of carbon nanostructures.

We have compared the present prediction results with existing ones from literatures [20-24]. Figure 8(a) and (b) show the stress-strain curves predicted by the various prediction methods as well as the present one for zigzag and armchair CNTs, respectively. It can be clearly seen that the present method yields similar prediction results in terms of both stiffness and strength as compared with the other methods based on MD and FE methods.

We also predicted the buckling behavior of the CNTs by applying the compressive loading along the CNT's axial direction. Figure 9 shows the stress-strain curve and the deformed shapes of a $(10,10)$ CNT with the length of $10 \mathrm{~nm}$ at various strain states under the compressive loading. The CNT is uniformly compressive in the axial direction until it starts to buckle to bend as shown in Figure 9(b). As shown in the stress-strain curve, the 
CNT is compressed nearly linearly until the buckling at $\sigma_{1}=123.0 \mathrm{GPa}$ and $\varepsilon_{1}=8.3 \%$. With the further compression, the $(10,10)$ CNT continues to buckling and forms hinges in two locations as shown in Figure 9(c)-(e). The maximum buckling stress is significantly dependent on the aspect ratio of the CNT. Figure 10 shows the stress-strain curve and the deformed shapes of a CNT with the same $(10,10)$ chirality but a longer length of $30 \mathrm{~nm}$. This longer CNT shows a simpler Euler-type buckling bent shape. The stress-strain plots comparing the two CNTs of $10 \mathrm{~nm}$ and $30 \mathrm{~nm}$ long show that as the CNT length increases, the maximum buckling stress decreases significantly.

Often, CNTs fabricated in the labs tend to have many defects, which would affect their properties significantly. In this study, we have applied the present method to evaluate the effects of CNT defects on the mechanical properties such as modulus and strength. A defected CNT was constructed with a semiconducting $(8,0)$ and metallic $(7,1)$ CNTs by fusing them at the junction, which forms a small-angle connection [29, 30]. Figure 11 shows the stress-strain curve and the deformed shapes of the $(8,0) /(7,1)$ CNT at various strain states under the tensile loading. To make smooth transition between the CNTs, the junction tends to form non-hexagonal C-C rings. As Figure 11 (b) shows, in this $(8,0) /(7,1)$ CNT case, the junction between these two CNTs consists of one pentagon and one heptagon C-C rings. We applied a uniaxial tensile load to the defected CNT. As Figure 11(c) and (d) show, the CNT junction with the defects initially flattens with the increase of the load until it results in the fracture at the defected junction. With further increase of load, the defected junction becomes the source of initiation of the damage, and results in the drastic stress drop, as shown in Figure 11(a) 
and (e)-(f). To evaluate the effect of defect on the mechanical properties, the stress-strain curve of the defected CNT are re-plotted in Figure 12 along with those of the pristine $(8,0)$ and $(7,1)$ CNTs in dotted lines. The comparison of the stress-strain plots of the defected $(8,0) /(7,1)$ CNT and pristine $(8,0)$ and $(7,1)$ CNTs shows that not only the initial slope (stiffness) but also the maximum fracture strength are significantly lowered mainly because of the defected junction. This simulation result supports the observation that why the experimentally measured stiffness and strength values of CNTs are significantly lowered than theoretically predicted values since the most CNTs fabricated in reality possess many defects.

The junction between the CNT and graphene in the 3-D junctioned PGN consists of several non-hexagonal $\mathrm{C}-\mathrm{C}$ rings. As seen in the case of the defected CNT, the non-hexagonal C-C rings tend to be the source of initiation of the damage and may result in the drastic stress drop. Furthermore, because of columnar nature of the PGN structures, they are susceptible to buckling damage subject to compressive loading. We take a representative unitcell of one of such 3-D CNT-graphene junctioned nanostructure, and evaluate its structural performance under tensile and compressive loading. Figure 13 shows the stress-strain curves of single-walled CNT grown on graphene sheet loaded under tension and compression loading. The stress-strain plot shows that the initial slopes for the tension and compression are similar with each other. However, these two modes of loading results in drastically different maximum strength values as well as post-failure behaviors. Note that the failure occurs near the CNT-graphene junctions in both cases due to the heptagonal rings around the junction. Note also that after this junctioned 
nanostructure buckles under the compression, the stress-strain curve becomes flattened, which is similar to a post-failure densification behavior of porous foam materials [31].

Figure 14 shows the stress-strain curve and the deformed shapes of a more ideal 3-D junctioned structure having periodicity in all three directions. The 3-D PGN subject to compressive loading in the pillar direction shows nearly linear deformation behavior until it starts to buckle, as Figure 14(b) and (c) show. The nearly linear increase in the stress with respect to the strain loading ends at $\varepsilon_{3}=11.1 \%$ when two graphene sheets slightly shift horizontally, as Figure 14(d) shows. With further increase of strain, the graphene sheets shift horizontally further while the CNT pillars remain nearly straight. The reason of this buckling mode of horizontal shift of the graphene sheets rather than the CNT pillars is due to significantly larger axial stiffness of the CNT pillars compared with the relatively flexible bending stiffness of the graphene sheets. Note that this horizontal shift mode of the graphene sheets predicted by the present nonlinear analysis coincides with the previous prediction by a structural molecular mechanics modeling based on the linear Euler buckling analysis [32].

The present prediction method combines the MM and MD simulations to obtain the global minimum of the energy states. As stated earlier, we implemented the MD simulations because the MM simulations could lead to the local energy minimum. Here, we compare the simulation results based on MM only and combined MM and MD simulations. We took a PGN structure as a representative case of complex carbon nanostructures. Figure 15 shows the stress-strain plot of a PGN structure under compression in the CNT pillar 
direction. The dotted line designated as "local minimum" was obtained with the MM simulations only without the MD simulations. Many spikes and wiggles during the load increase indicate unreliable prediction of the minimal energy states, as compared with the smooth curve predicted by the combined method. Therefore, we can conclude that the MD simulations are an essential step to obtain the global energy minimum, which leads to reliable strength prediction for such nanostructures with complex geometries..

\section{Summary and Conclusions}

We have developed the computational scheme to predict stiffness and strength of carbon nanostructures under various loading modes. The prediction method is based on combined MM and MD simulations by ensuring the global energy minimum at a given loading level. The MM simulations enable us to conduct the simulations with reasonably large load increment, while the MD simulations help us in finding the global energy minimum at each load step. The significance of finding the global energy minimum by using the combined MM and MD simulations are pronounced with more complicated 3-D nanostructures such as PGN.

We have applied the present method to various carbon nanostructures including 1-D CNTs, 2-D graphene, CNT with defects, 3-D CNT-graphene junctioned PGN. We have identified the maximum stress and strain at failure of these carbon nanostructures as well as their critical failure modes. The present prediction method can be utilized to further predict other carbon nanostructures which were not handled in this paper, including multi-walled carbon nanotubes, multi-layered graphites, CNT ropes and bundles, CNT 
yarns, etc.

\section{Acknowledgments}

This work was financially supported by U.S. Air Force Research Laboratory and Air Force Office of Scientific Research with Contract Nos. FA865010-D-5011 and FA8650-07-D-5800-45, respectively.

\section{References}

\section{References}

[1] E. W. Wong, P. E. Sheehan, C. M. Lieber, Nanobeam mechanics: Elasticity, strength, and toughness of nanorods and nanotubes, Science 277 (5334) (1997) 1971-1975.

[2] S. Frank, P. Poncharal, Z. L. Wang, W. A. d. Heer, Carbon nanotube quantum resistors, Science 280 (5370) (1998) 1744-1746.

[3] S. Berber, Y.-K. Kwon, D. Tománek, Unusually high thermal conductivity of carbon nanotubes, Physical Review Letters 84 (20) (2000) 46134616.

[4] A. V. Krasheninnikov, F. Banhart, Engineering of nanostructured carbon materials with electron or ion beams, Nat Mater 6 (10) (2007) $723-733$.

[5] M. Terrones, F. Banhart, N. Grobert, J. C. Charlier, H. Terrones, P. M. Ajayan, Molecular junctions by joining single-walled carbon nanotubes, Physical Review Letters 89 (7) (2002) 075505. 
[6] C. Jin, K. Suenaga, S. Iijima, Plumbing carbon nanotubes, Nat Nano 3 (1) (2008) 17-21.

[7] J.-M. Ting, C.-C. Chang, Multijunction carbon nanotube network, Applied Physics Letters 80 (2) (2002) 324-325.

[8] V. Varshney, S. S. Patnaik, A. K. Roy, G. Froudakis, B. L. Farmer, Modeling of thermal transport in pillared-graphene architectures, ACS Nano 4 (2) (2010) 1153-1161.

[9] S. Sihn, V. Varshney, A. K. Roy, B. L. Farmer, Prediction of 3D elastic moduli and poisson's ratios of pillared graphene nanostructures, Carbon 50 (2) (2012) 603-611.

[10] S. Iijima, C. Brabec, A. Maiti, J. Bernholc, Structural flexibility of carbon nanotubes, The Journal of Chemical Physics 104 (5) (1996) 20892092.

[11] B. I. Yakobson, M. P. Campbell, C. J. Brabec, J. Bernholc, High strain rate fracture and $\mathrm{C}$-chain unraveling in carbon nanotubes, Computational Materials Science 8 (4) (1997) 341-348.

[12] E. Hernández, C. Goze, P. Bernier, A. Rubio, Elastic properties of C and BxCyNz composite nanotubes, Physical Review Letters 80 (20) (1998) 4502-4505.

[13] D. Sánchez-Portal, E. Artacho, J. M. Soler, A. Rubio, P. Ordej, Ab initio structural, elastic, and vibrational properties of carbon nanotubes, Physical Review B 59 (19) (1999) 12678-12688. 
[14] C. Q. Ru, Effective bending stiffness of carbon nanotubes, Physical Review B 62 (15) (2000) 9973-9976.

[15] C. Q. Ru, Elastic buckling of single-walled carbon nanotube ropes under high pressure, Physical Review B 62 (15) (2000) 10405-10408.

[16] Y. J. Liu, X. L. Chen, Evaluations of the effective material properties of carbon nanotube-based composites using a nanoscale representative volume element, Mechanics of Materials 35 (1-2) (2003) 69-81.

[17] C. Li, T.-W. Chou, A structural mechanics approach for the analysis of carbon nanotubes, International Journal of Solids and Structures 40 (10) (2003) 2487-2499.

[18] C. Li, T.-W. Chou, Elastic moduli of multi-walled carbon nanotubes and the effect of van der Waals forces, Composites Science and Technology 63 (11) (2003) 1517-1524.

[19] A. Ghavamian, M. Rahmandoust, A. Öchsner, "a numerical evaluation of the influence of defects on the elastic modulus of single and multiwalled carbon nanotubes, Computational Materials Science 62 (2012) 110-116.

[20] T. Belytschko, S. P. Xiao, G. C. Schatz, R. S. Ruoff, Atomistic simulations of nanotube fracture, Physical Review B 65 (23) (2002) 235430.

[21] M. Meo, M. Rossi, Tensile failure prediction of single wall carbon nanotube, Engineering Fracture Mechanics 73 (17) (2006) 2589-2599. 
[22] M. Meo, M. Rossi, A molecular-mechanics based finite element model for strength prediction of single wall carbon nanotubes, Materials Science and Engineering: A 454-455 (2007) 170-177.

[23] K. I. Tserpes, P. Papanikos, S. A. Tsirkas, A progressive fracture model for carbon nanotubes, Composites Part B: Engineering 37 (7-8) (2006) 662-669.

[24] J. R. Xiao, J. Staniszewski, J. W. Gillespie Jr, Fracture and progressive failure of defective graphene sheets and carbon nanotubes, Composite Structures 88 (4) (2009) 602-609.

[25] S. Plimpton, Fast parallel algorithms for short-range molecular dynamics, Journal of Computational Physics 117 (1) (1995) 1-19.

[26] H. Sun, S. J. Mumby, J. R. Maple, A. T. Hagler, An ab initio CFF93 allatom force field for polycarbonates, Journal of the American Chemical Society 116 (7) (1994) 2978-2987.

[27] B. H. Park, Some useful math for molecular mechanics, Tech. rep. (June 17 2002).

[28] http://lammps.sandia.gov/doc/compute_pressure.html.

[29] L. Chico, V. H. Crespi, L. X. Benedict, S. G. Louie, M. L. Cohen, Pure carbon nanoscale devices: Nanotube heterojunctions, Physical Review Letters 76 (6) (1996) 971-974.

[30] V. Meunier, L. Henrard, P. Lambin, Energetics of bent carbon nanotubes, Physical Review B 57 (4) (1998) 2586-2591. 
[31] L. J. Gibson, M. F. Ashby, Cellular Solids: Structure and Properties, Cambridge University Press, New York, 1988.

[32] S. Sihn, V. Varshney, A. K. Roy, Modeling of mechanical behavior of pillared graphene structures, in: The 51st AIAA/ASME/ASCE/AHS/ASC Structures, Structural Dynamics, and Materials Conference, Orlando, FL, 2010. 


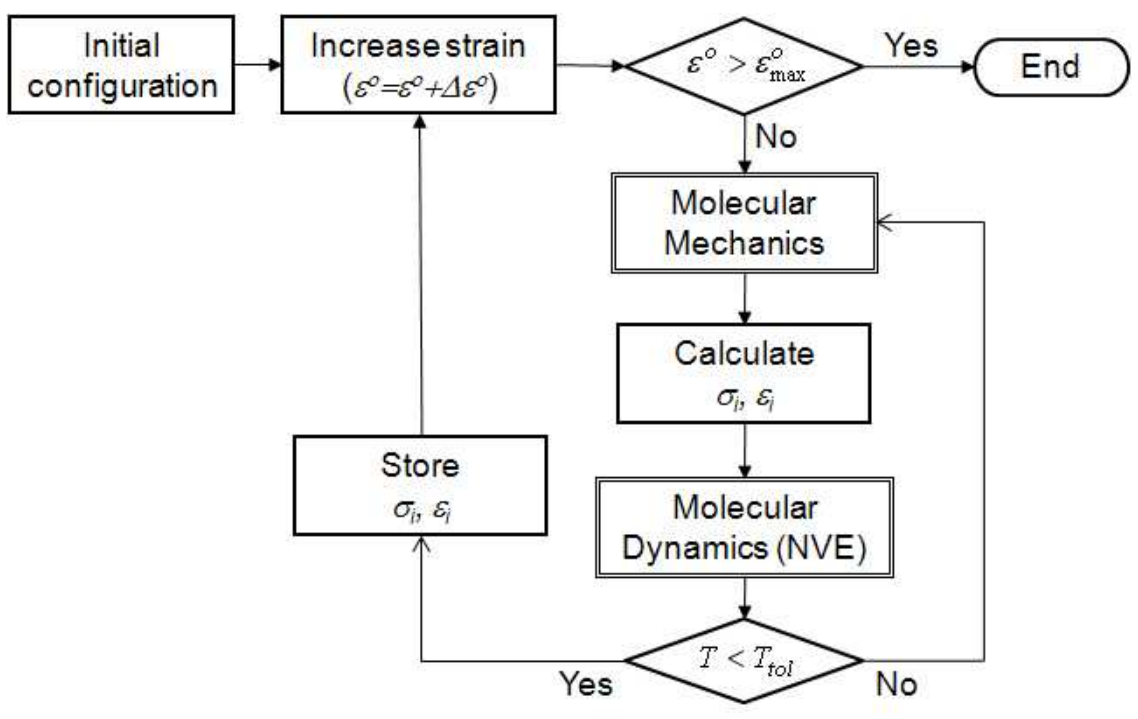

Figure 1: Strength prediction analysis scheme for carbon nanostructures.
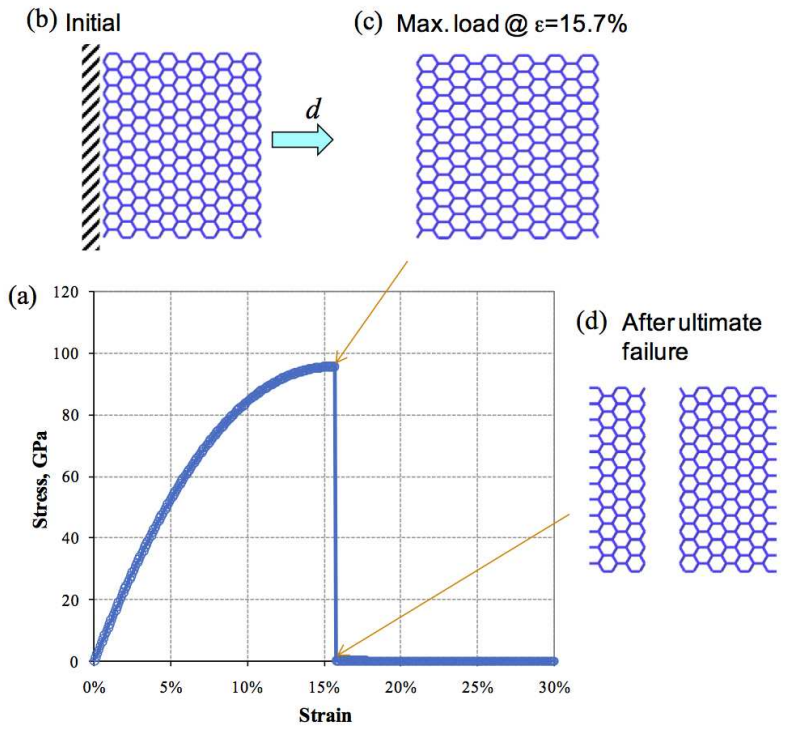

Figure 2: Graphene layer under tensile loading in $x$-direction. 


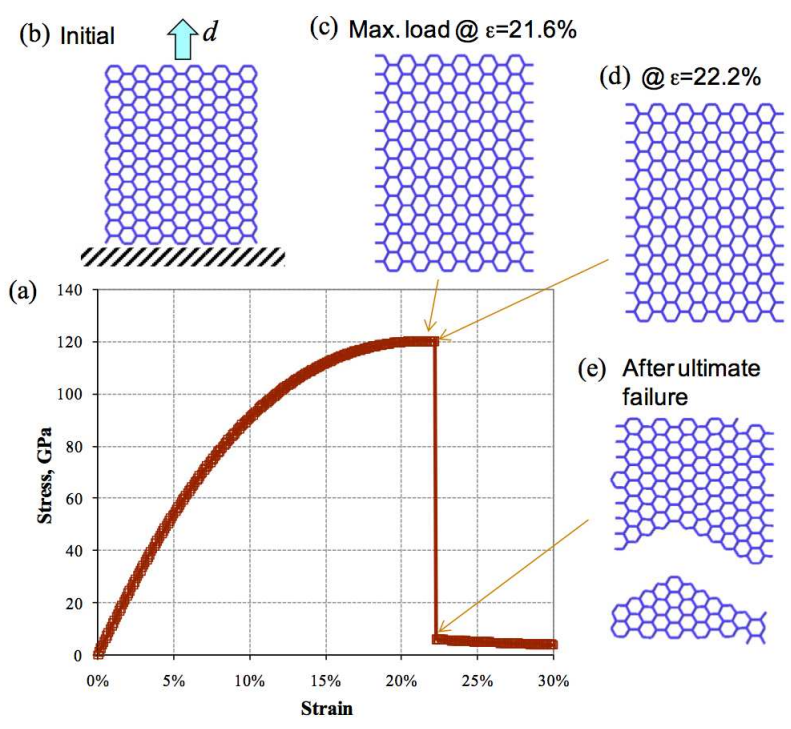

Figure 3: Graphene layer under tensile loading in $y$-direction.

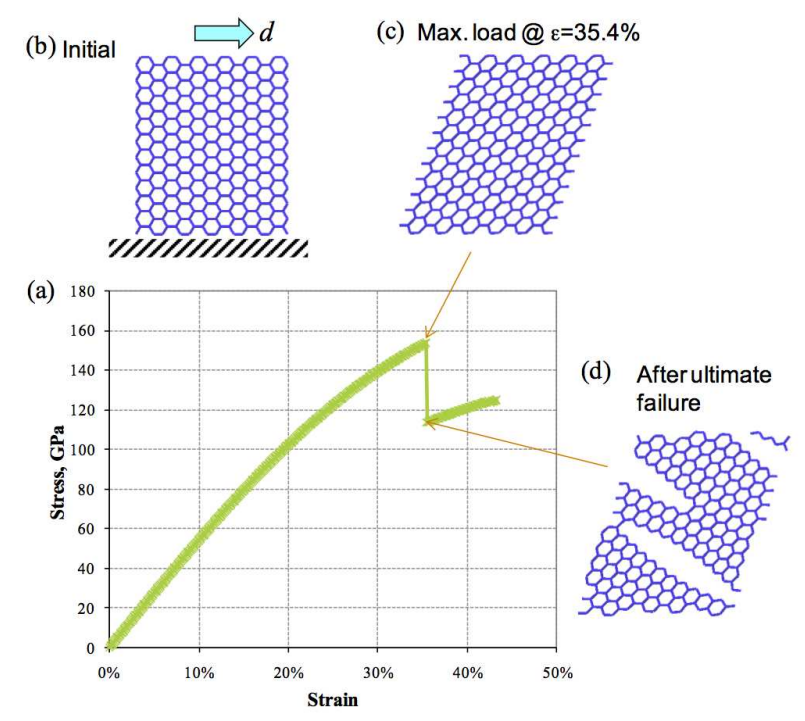

Figure 4: Graphene layer under shear loading. 


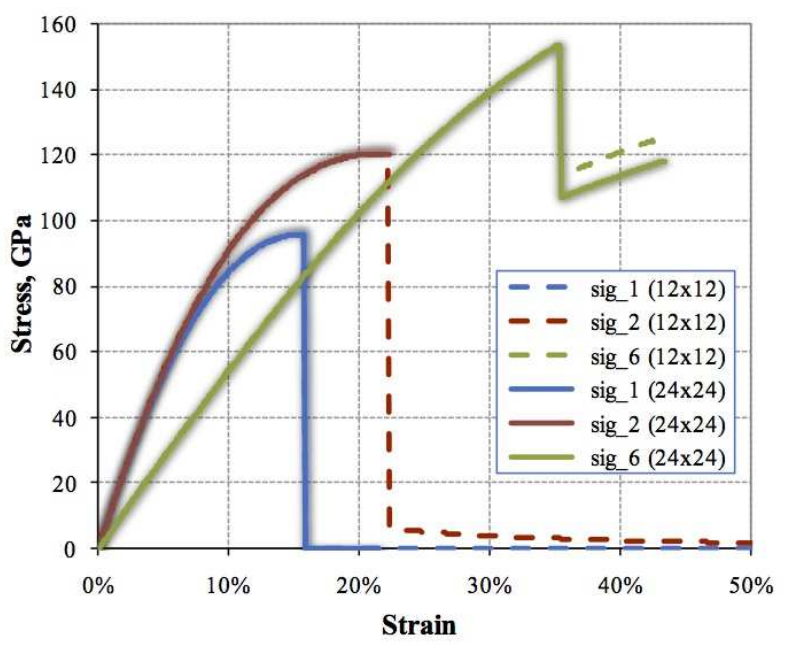

Figure 5: Stress-strain curves of graphene sheets of two sizes $(12 \times 12$ and $24 \times 24)$ under axial tensile loads $\left(\sigma_{1}\right.$ and $\left.\sigma_{2}\right)$ and a shear load $\left(\sigma_{6}=\tau_{12}\right)$. 


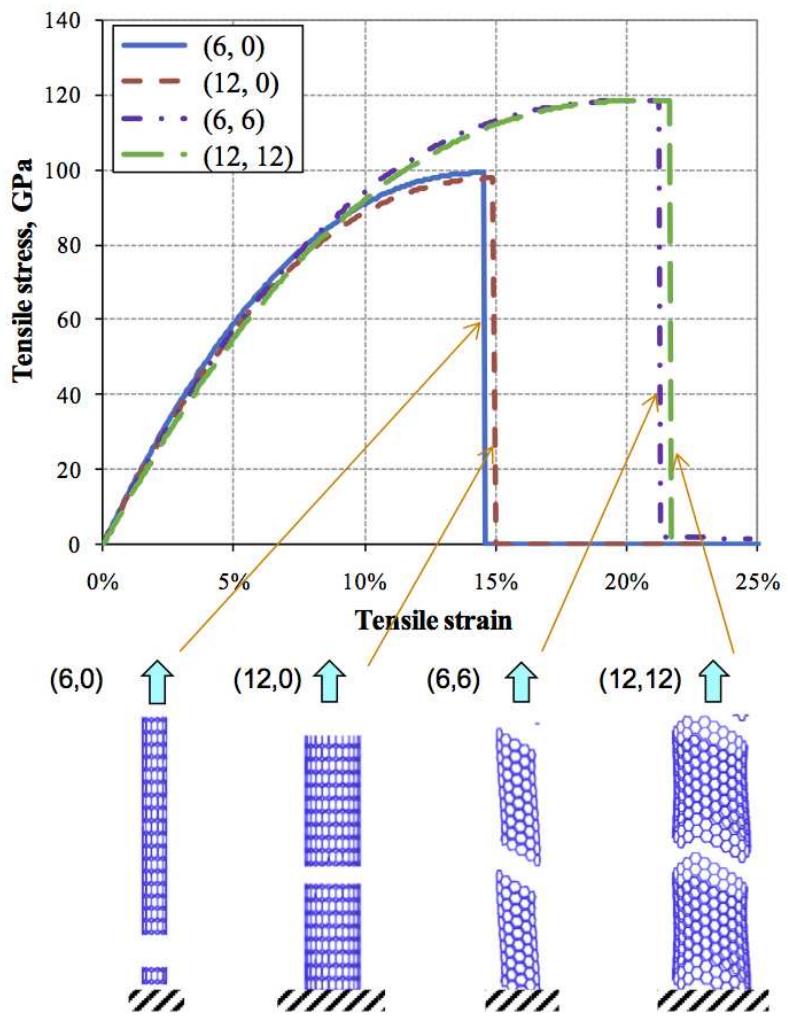

Figure 6: CNT with four different chiralities under axial tensile loading. 


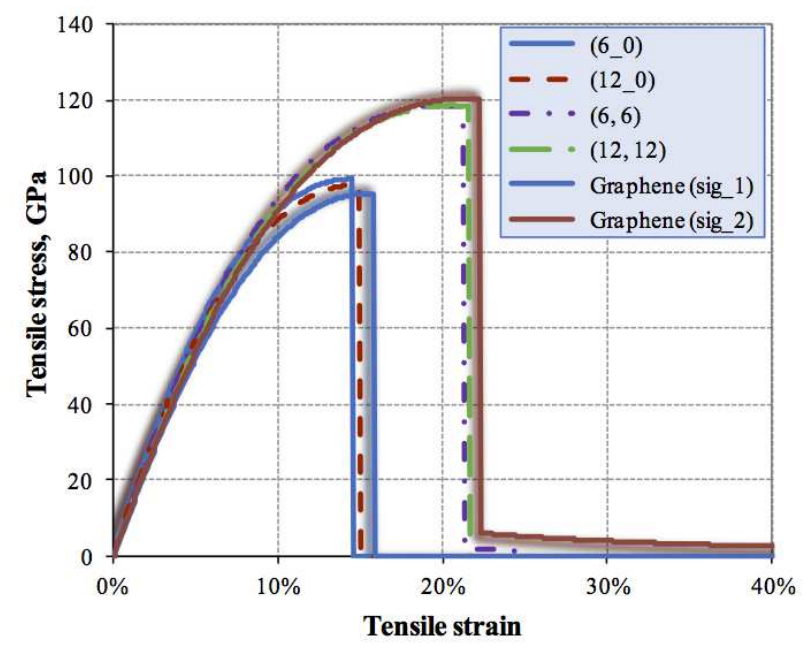

Figure 7: Comparison of stress-strain curves of graphene sheets and CNTs under tensile loading.

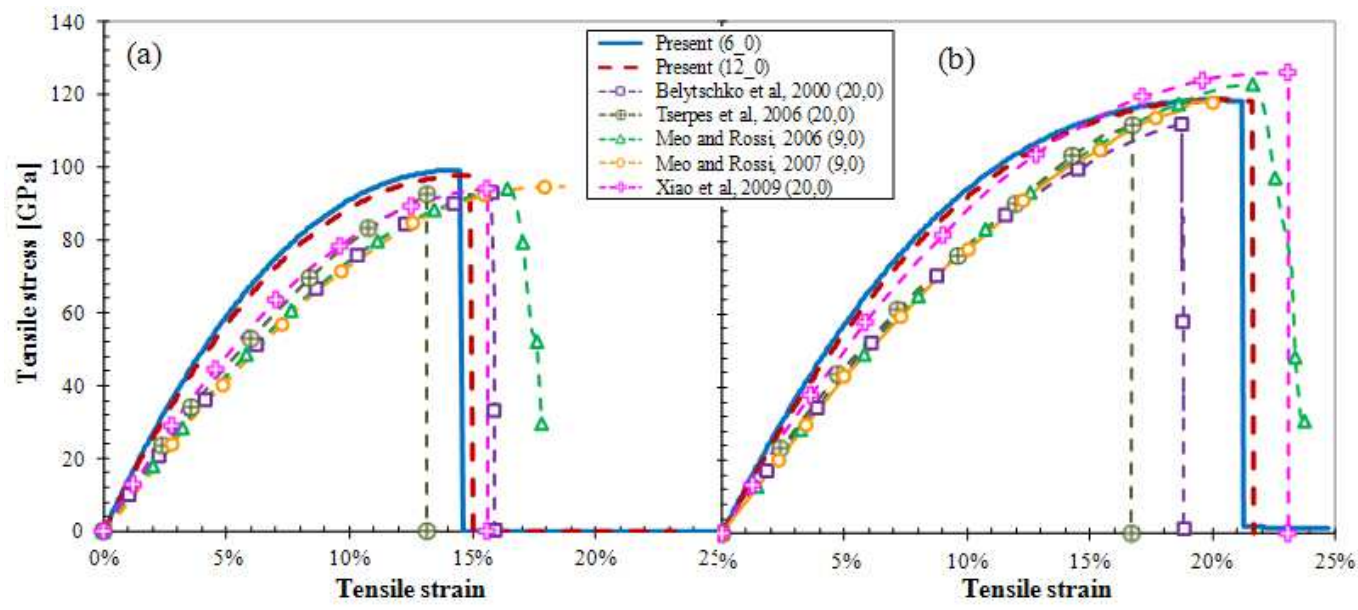

Figure 8: Comparison of various prediction methods: (a) zigzag CNTs, (b) armchair CNTs. 


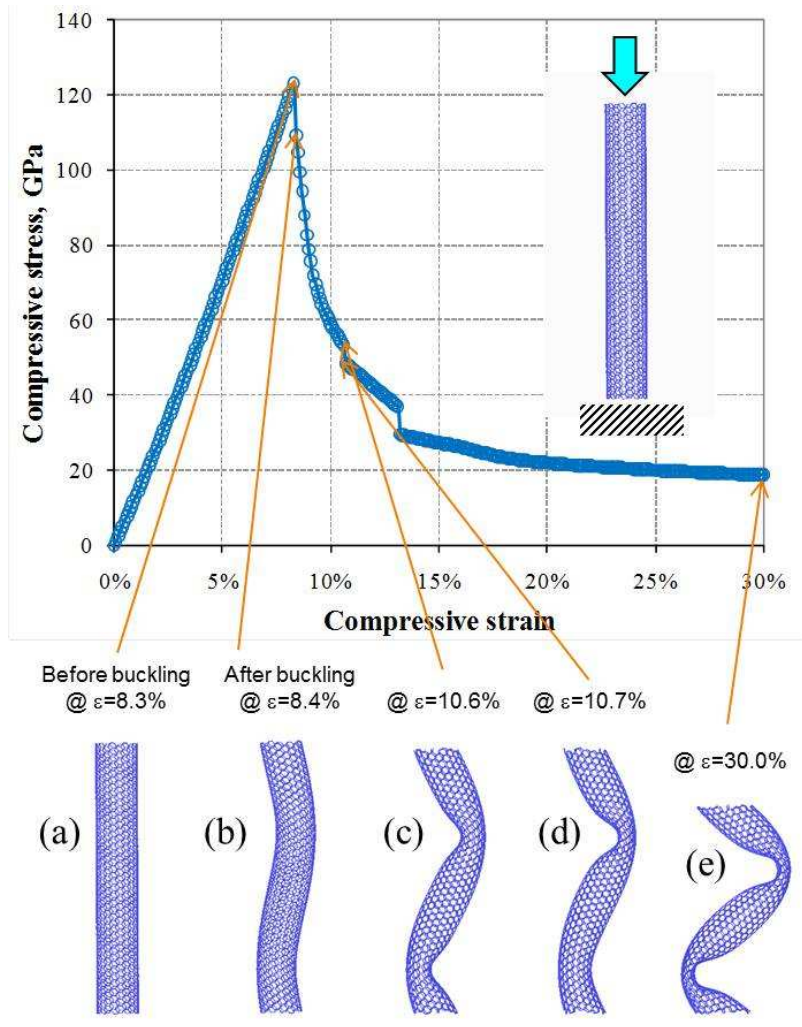

Figure 9: $(10,10) \mathrm{CNT}$ with the length of $10 \mathrm{~nm}$ under compressive loading. 


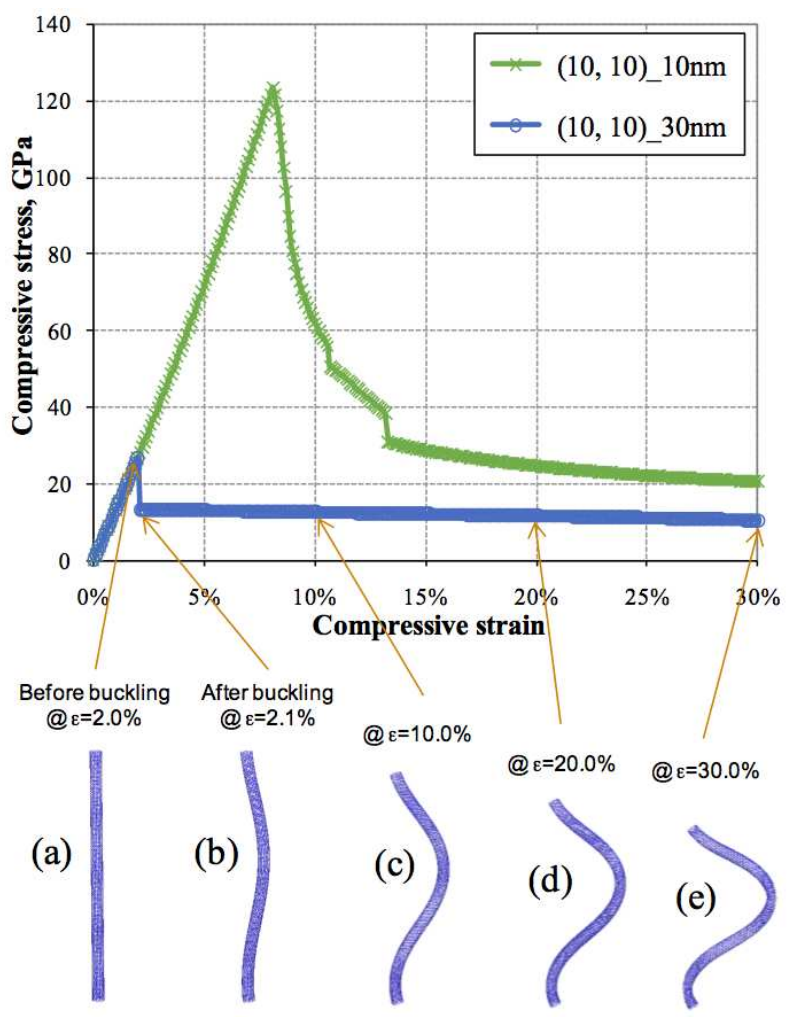

Figure 10: $(10,10)$ CNT with the length of $30 \mathrm{~nm}$ under compressive loading. 


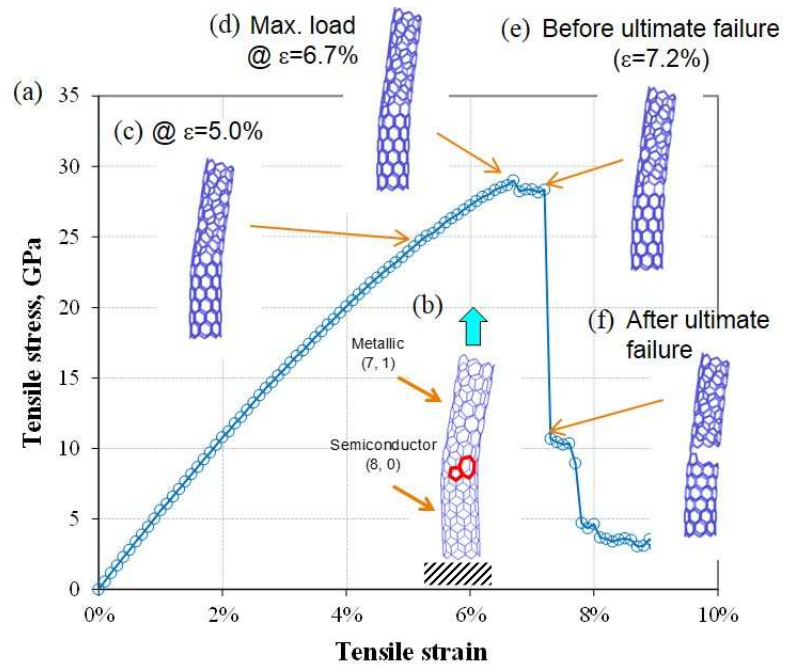

Figure 11: A CNT with a defected junction under tensile loading.

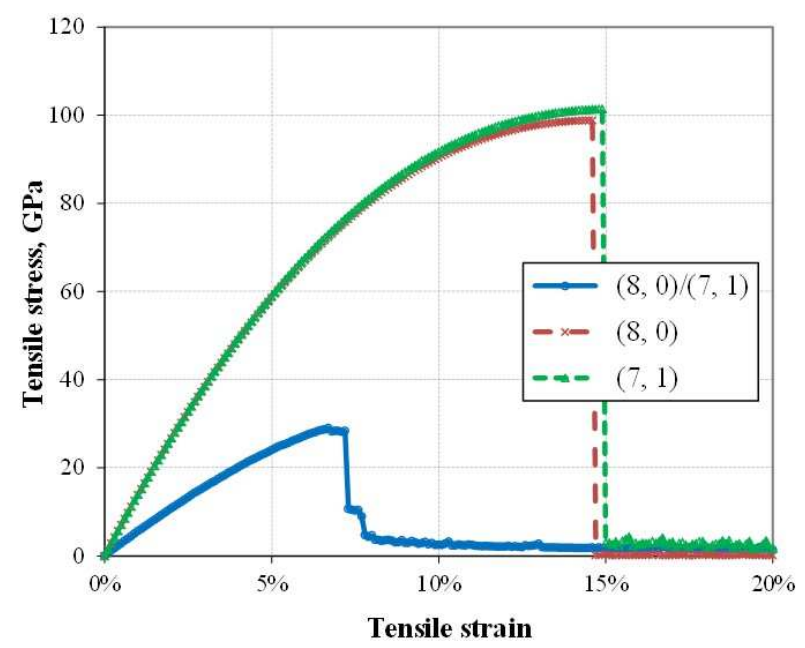

Figure 12: Comparison of the stress-strain plots of the defected $(8,0) /(7,1)$ CNT and pristine $(8,0)$ and $(7,1)$ CNTs. 


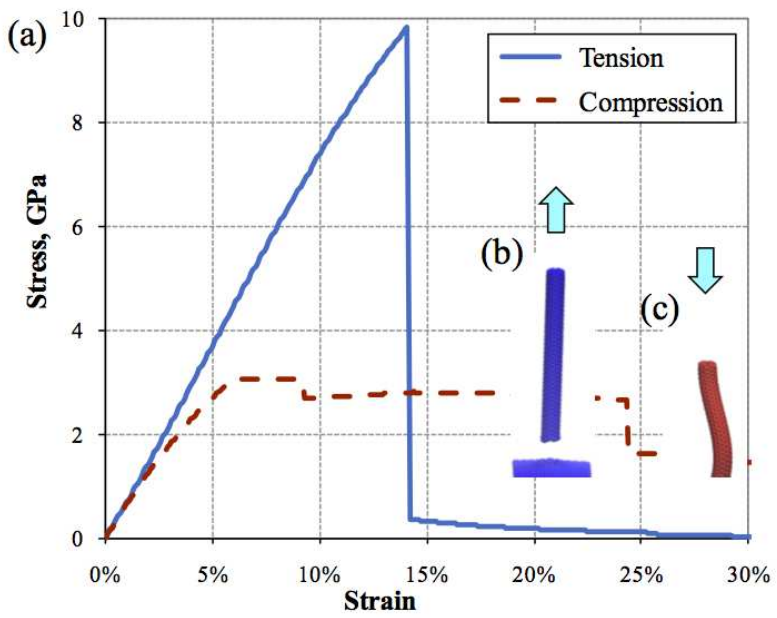

Figure 13: A CNT-graphene junctioned 3-D carbon nanostructure under tensile and compressive loading. 

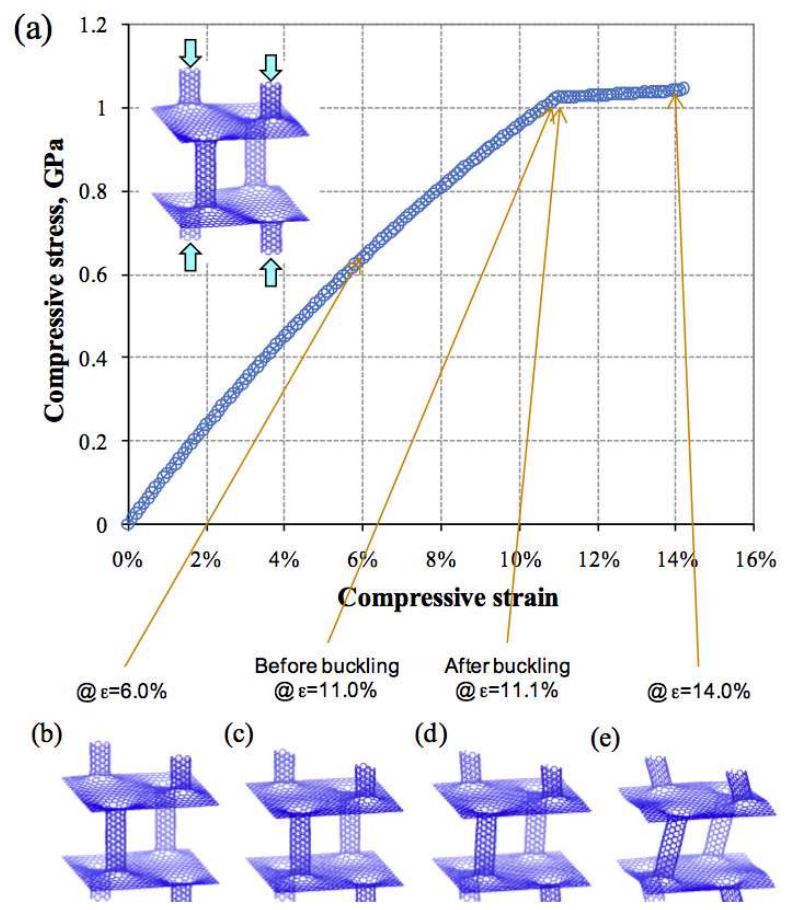

Figure 14: A pillared graphene nanostructure under compressive loading. 


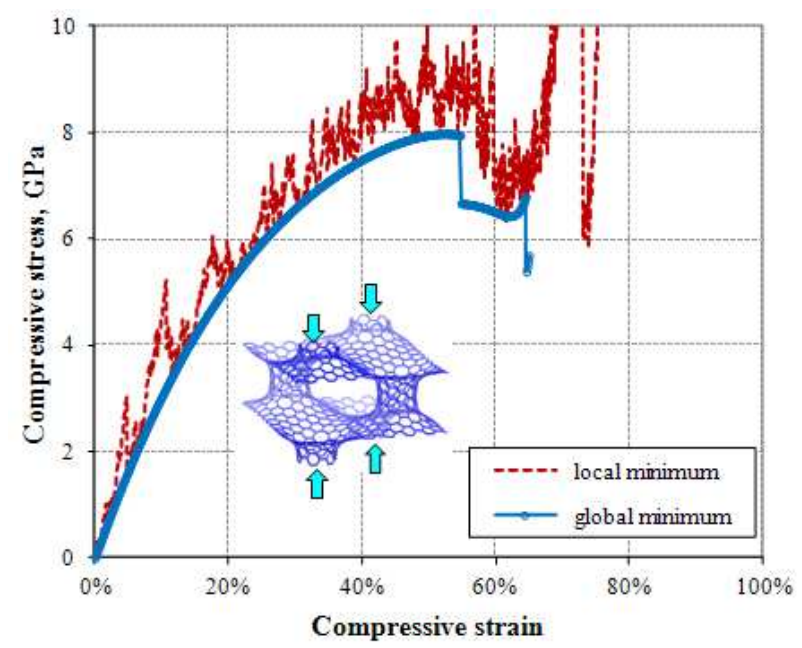

Figure 15: Stress-strain behaviors of a pillared graphene nanostructure under compressive loading predicted with local and global energy minima. 